Part of Journal of Research of the National Bureau of Standards, Volume 17, November 1936

\title{
ESTIMATION OF CHROMATICITY DIFFERENCES AND NEAREST COLOR TEMPERATURE ON THE STANDARD 1931 ICI COLORIMETRIC COORDINATE SYSTEM
}

\author{
By Deane B. Judd
}

\section{ABSTRACT}

Estimation of chromaticity differences has been facilitated by the preparation of a standard mixture diagram showing by a group of ellipses the scale of perceptibility at the various parts of the diagram. The distances from the boundaries of the ellipses to their respective "centers" all correspond approximately to the same number (100) of "least perceptible differences."

The estimation of nearest color temperature has been facilitated by the preparation of a mixture diagram on which is shown a family of straight lines intersecting the Planckian locus; each straight line corresponds approximately to the locus of points representing stimuli of chromaticity more closely resembling that of the Planckian radiator at the intersection than that of any other Planckian radiator.

\section{CONTENTS}

1. Tntroduction $\ldots \ldots \ldots$

II. Estimation of chromaticity differences

III. Nearest color temperature

\section{INTRODUCTION}

Since the adoption, in 1931, by the International Commission on Illumination of a standard colorimetric coordinate system, by far the majority of fundamental colorimetric specifications have been expressed in that system. ${ }^{1}$ A minor defect which is coming to be more keenly felt as the use of the system becomes more extended is the difficulty of interpreting differences in position on the standard mixture diagram in terms of degree and kind of chromaticity difference. The discovery of a projective transformation of the standard system whose Maxwell triangle yields nearly uniform chromaticity scales ${ }^{2}$ is a step toward remedying this defect; on this triangle the length of any line is approximately proportional to the perceptibility of the chromaticity difference between the stimuli represented at the extremes of the line.

1 D. B. Judd, The 1931 ICI standard observer and coordinate system for colorimetry, J. Opt. Soc. Am. 23, 359 (1933).

${ }^{2}$ D. B. Judd, A Maxwell triangle yielding uniform chromaticity scales, J. Research NBS 14, 41 (1935) RP75f; also J. Opt. Soc. Am. 25, 24 (1935).

$99074-36-10$ 
The substitution of the uniform-chromaticity-scale (UCS) system for the ICI system immediately suggests itself, but there are several reasons why this should not be done at present. In the first place, although the UCS system was derived from and is a valid representation of about nine-tenths of the available data on sensibility to chromaticity differences, and although, furthermore, many divergent applications of it $^{3}$ have shown it to be a definite improvement in spacing over the ICI system, nevertheless the original data are neither sufficiently numerous nor sufficiently self-consistent to be considered definitely final. It is probable that further important improvements in spacing can be made. A second reason for adherence to the present standard ICI system is that the ICI system is more suited to the representation of photometric quantities; this may be seen from the fact that the ICI luminosity coefficients are $0,1,0$, while the UCS luminosity coefficients are $0.08852,0.11112,-0.09802$. A third reason is that the applicability of data obtained by observing small apertures to observation of chromaticity differences between extended surface colors has not been demonstrated, nor have these data on small differences been shown to apply by simple addition to large chromaticity differences. The optimistic view that such applicability does not require demonstration has been fairly general, but extensive data which are being accumulated do not wholly justify this view. They indicate that the UCS triangle is applicable to small chromaticity differences between surface colors of large as well as small extent; furthermore, the application of the UCS triangle to large as well as small chromaticity differences between point sources (signal lights) fails to result in any discrepancies not explainable by aberrations of the lens system of the eye; but there are data which suggest that the UCS triangle is not applicable to large chromaticity differences between extended surfaces. For these three reasons no departure from the standard 1931 ICI system is proposed at this time.

Accordingly, then, for research purposes many determinations in the colorimetry section of the National Bureau of Standards are being recorded in duplicate, once in the ICI system and once in the UCS system; but there are also many determintions which are expressed in the ICI system alone. For such determinations some of the advantages of the UCS system may be obtained if there is available a graphical representation of the relative scales of the two systems. The present paper serves to give two such representations; one of them shows how the scale of perceptibility varies over the ICI mixture diagram on the assumption that the UCS system gives accurately uniform chromaticity scales as it was intended to do, the other shows, on the same assumption, isotemperature lines to aid in the estimation of nearest color temperature for near-Planckian colors. ${ }^{4}$

\footnotetext{
3 See, for example, D. B. Judd, A method for determining the whiteness of paper, Paper Trade J. 100, no. 21 (1935) TS40; also Tech. Assn. Pap. [18] 392 (1935); and D. B. Judd, A method for determining whitenes of paper, II, Tech. Assn. Pap., [19] 359 (1935).

1 For these terms and concepts, see R. Davis, A correlated color temperature for illuminants, BS J. Research \%, 659 (1931) RP365.
} 


\section{ESTIMATION OF CHROMATICITY DIFFERENCES}

The equilateral Maxwell triangle of the ICI system, and all three of the mixture diagrams obtained by plotting in rectangular coordinates one of the ICI trilinear coordinates $(x, y, z)$ against another, fail to array color stimuli in such a way that equal chromaticity differences are indicated by lines of equal length. It is therefore impossible to give any single reliable perceptibility scale of chromaticity which will apply to the whole diagram as a scale of miles applies to a map.

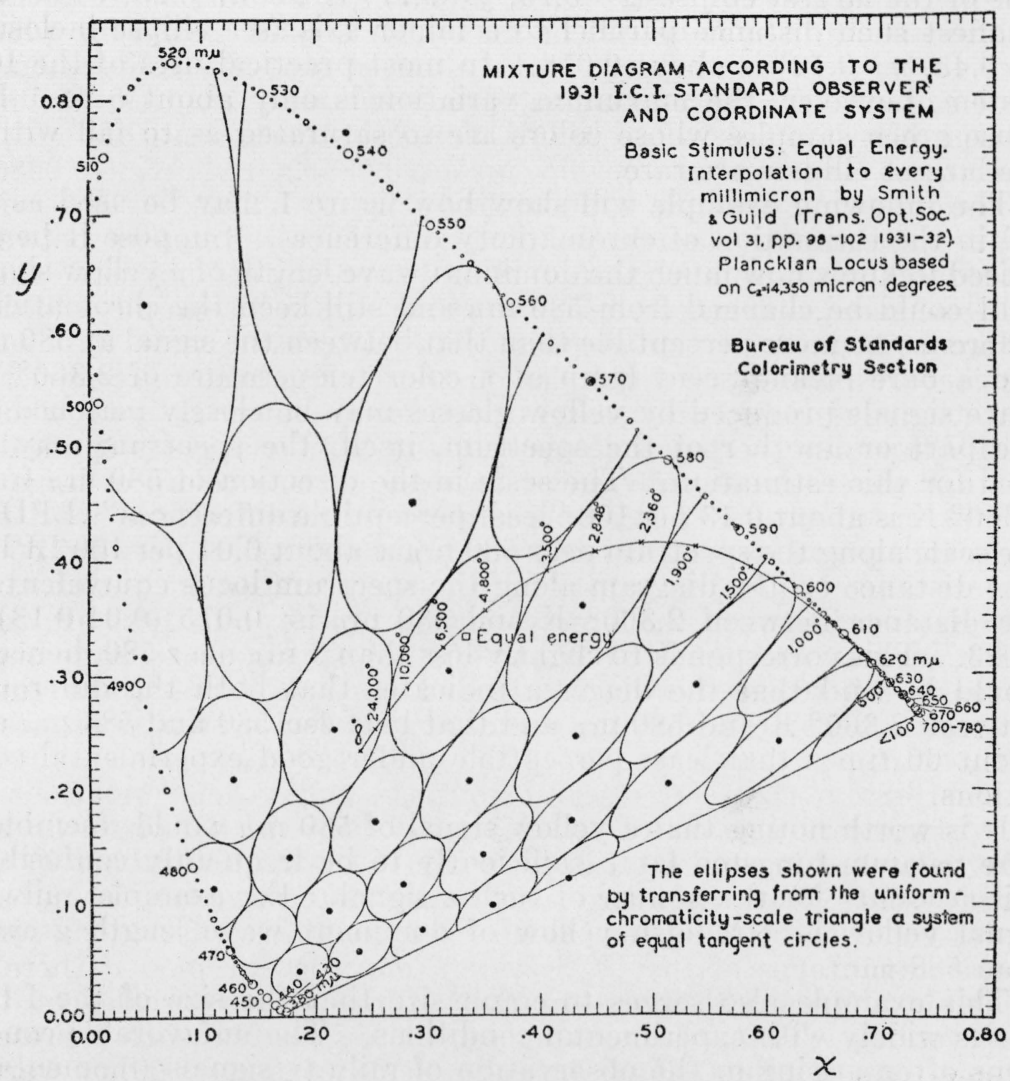

FIGURE 1.-Perceptibility scales for the standard 1931 ICI colorimetric coordinate system

The distances from points on the boundary of each ellipse to the indieated point within it all correspond approximately to 100 times the chromaticity difference just perceptible with certainty under moderately good observing conditions.

Instead of a single perceptibility scale there is required for a given direction on the mixture diagram a different scale for each different portion of the diagram, and for each point a different scale is, in general, required for each direction. In an effort to provide such a complete set of approximate perceptibility scales for the ICI mixture diagram a group of thirty tangent circles on the UCS system have been transferred to the $(x, y)$-plot of the ICI system where they appear as tangent ellipses of various sizes, eccentricities, and orientations; see figure 1. The distances from points on the boundaries of 
these ellipses to the point within them representing the transformed centers of the original circles give the desired scales of perceptibility; one one-hundredth of each such distance refers to a chromaticity difference just perceptible with certainty under moderately good experimental conditions (careful monocular observation of a $6^{\circ}$ circular field divided along a diameter yielding retinal illuminations between 200 and 1,000 photons).

The indicated maximum variation in the scales of perceptibility is about 14 to 1 ; the distance from point to boundary along the major axis of the largest ellipse $(x=0.23, y=0.77)$ is about 0.25 , while the smallest such distance parallel to a minor axis (see ellipse inclosing $x=0.43, y=0.12$ ) is about 0.018 . In most practical uses of the ICI system, however, the maximum variation is only about 5 to 1 because green samples whose colors are so saturated as to fall within the largest ellipses are rare.

The following example will show how figure 1 may be used as an aid in the estimation of chromaticity differences. Suppose it be required to know how much the dominant wave length of a yellow signal light could be changed from $580 \mathrm{~m} \mu$ and still keep the chromaticity difference no more perceptible than that between the signal at $580 \mathrm{~m} \mu$ and a bare incandescent lamp at a color temperature of $2,360^{\circ} \mathrm{K}$. Since signals produced by yellow glasses may be closely matched by one part or another of the spectrum, itself, the spectrum may be used for this estimation. The scale in the direction of $580 \mathrm{~m} \mu$ from $2,360^{\circ} \mathrm{K}$ is about 0.13 per 100 "least perceptible differences" (LPDs); the scale along the spectrum near $580 \mathrm{~m} \mu$ is about 0.04 per 100 LPDs. The distance on the diagram along the spectrum locus equivalent to the distance between $2,360^{\circ} \mathrm{K}$ and $580 \mathrm{~m} \mu$ is: $0.075(0.04 / 0.13)=$ 0.023 . This corresponds to slightly less than $3 \mathrm{~m} \mu$ near 580 , hence it would be said that the diagram indicates that both the difference between $2,360^{\circ} \mathrm{K}$ and $580 \mathrm{~m} \mu$ and that between 580 and $583 \mathrm{~m} \mu$ are about 60 times that least perceptible under good experimental conditions.

It is worth noting that a yellow signal of $580 \mathrm{~m} \mu$ would resemble a bare vacuum-tungsten lamp sufficiently to be frequently confusible; this accounts for the nonuse of such a signal. For example, railway signal yellow is a reddish yellow of dominant wave length greater than $588 \mathrm{~m} \mu .^{5}$

This example also serves to emphasize that the size of the LPD varies widely with experimental conditions. The unfavorable conditions often arising in the observation of railway signals (momentary viewing of point source at a retinal illumination of less than 100 photons with no comparison light) require perhaps 50 to 100 times as large chromaticity differences for discrimination as are required under good laboratory conditions.

\section{NEAREST COLOR TEMPERATURE}

An important practical application of the UCS system is its use in finding from any series of colors the one most resembling any neighboring color of the same brilliance. In this system the method, of course, is to draw the shortest line from the locus of the series to the

${ }^{5}$ Association of American Railroads, Signal Section Specification 69-35, Signal glasses (exclusive of hand lantern globes). Manual of Signal Section, AAR, part 136 (1935). 
point representing the neighboring color. This is the geometric equivalent of the method of observation on which many plans of color grading are based. The characteristic of these plans is that they pay attention to chromaticity differences along a series of color standards, and in the usual case that no member of the series gives a perfect chromaticity match, the nearest match is set and the residual difference neglected. In this way a problem which, strictly, is twodimensional finds a one-dimensional solution that is close enough to be of practical use.

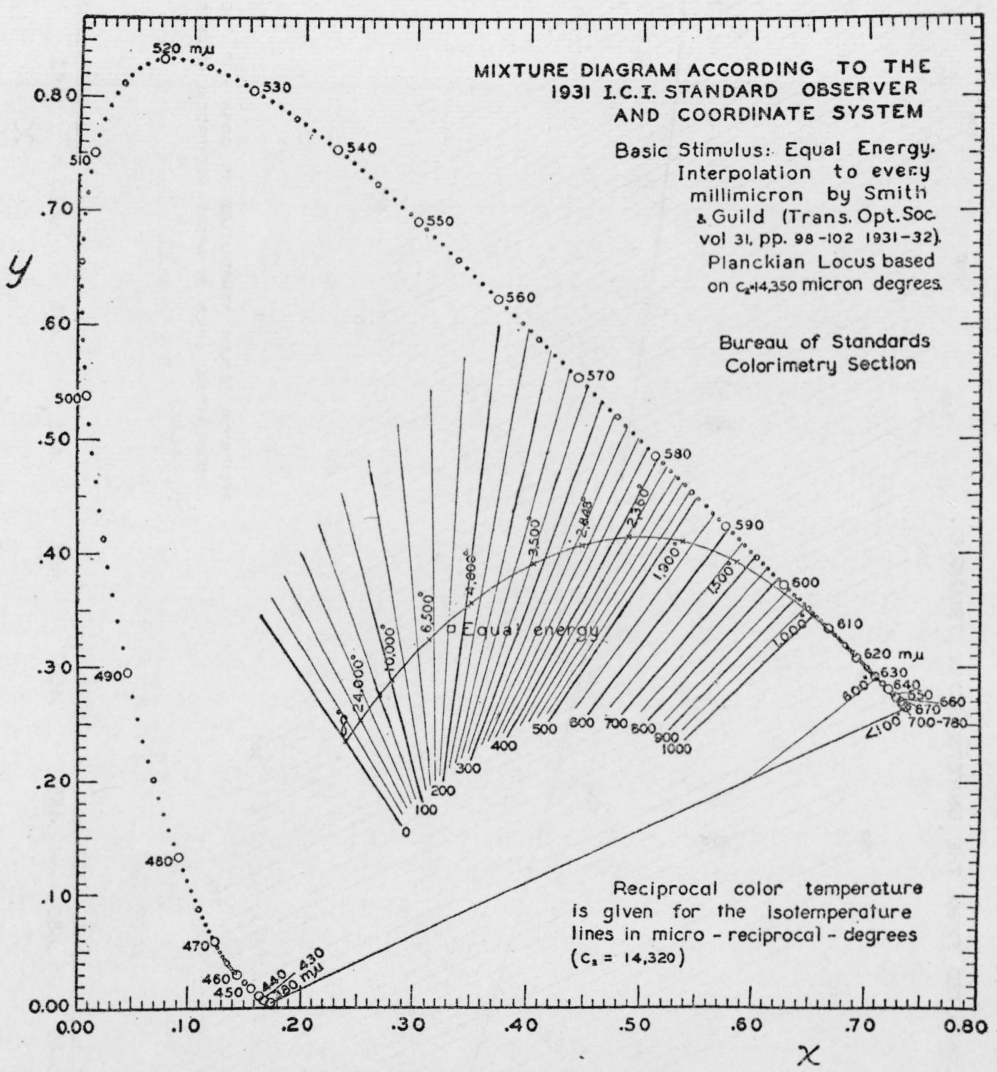

FIGURe 2.-Iso-temperature lines shown on the standard 1931 ICI colorimetric co. ordinate system.

Color stimuli specified on an iso-temperature line give closer chromaticity matches with the indicated Planckian color than with Planckian colors at neighboring color temperatures.

In finding nearest color from the series the UCS system may be used directly. The locus of the series is plotted together with the point representing the neighboring chromaticity, and the shortest line through the point to the locus, usually the normal, gives the nearest chromaticity of the series. It is also possible to carry out the operation once for all by drawing on the UCS triangle a number of normals to the locus of the series completely covering at sufficiently small intervals the areas of the triangle near the locus. These normals can 


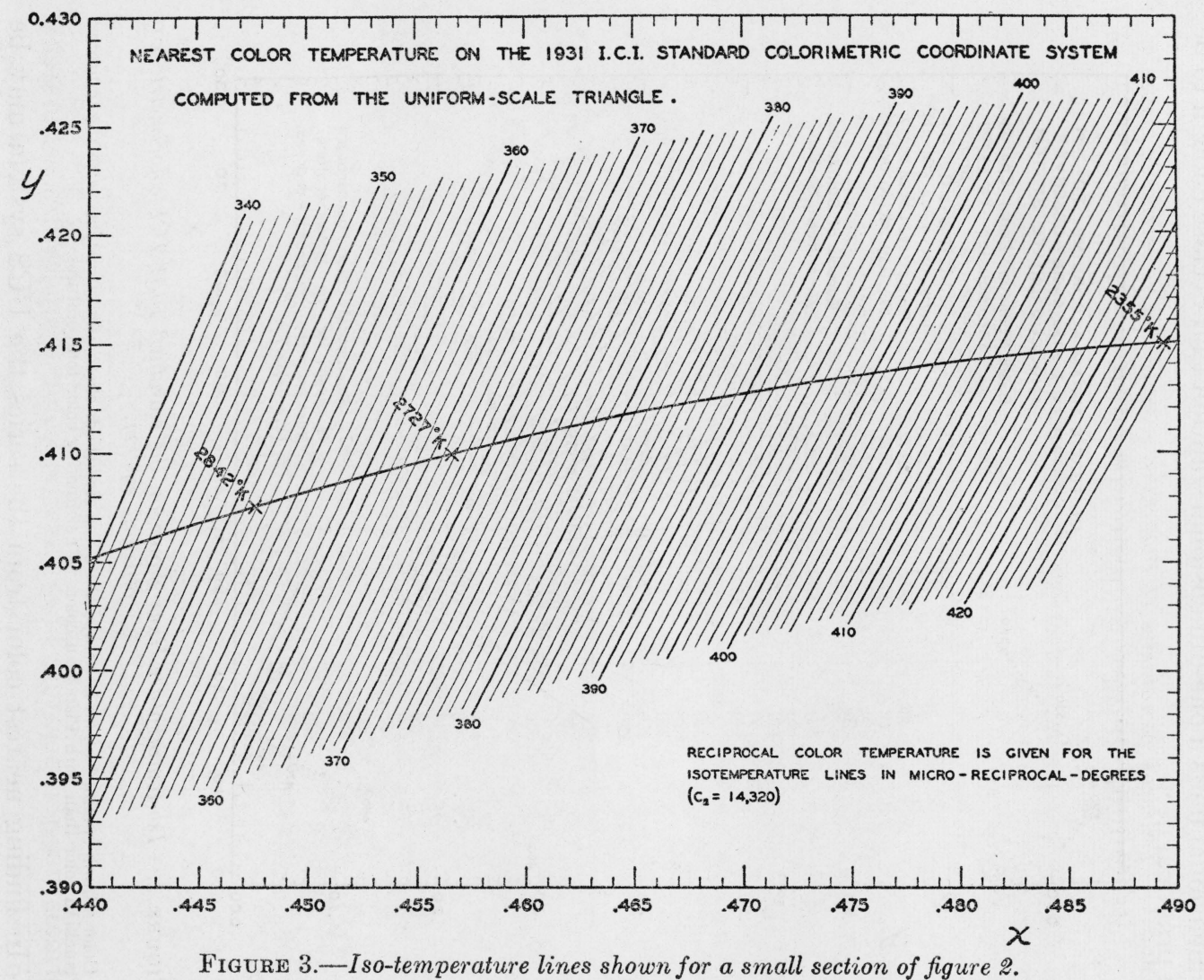

ฮี

है

This region includes the most-used portion of the color-temperature scale for incandescent lamps. 
then be transformed to any other desired system and the determination of nearest chromaticity carried on entirely outside the UCS system. This has been done by McNicholas in the estimation of nearest chromaticity of vegetable oils on both the wave-length scale and on the scale composed of combinations of Lovibond 35-yellow with various Lovibond red glasses. ${ }^{6}$ Figure 2 shows a similar series of normals to the Planckian locus transferred to the standard, 1931 ICI system. ${ }^{7} \quad$ By means of these transferred

TABLE 1.- Trilinear coordinates of Planckian stimuli and reciprocal of slopes of the iso-temperature lines as a function of reciprocal color temperature

\begin{tabular}{|c|c|c|c|}
\hline \multirow{2}{*}{$\begin{array}{c}1 / \theta \\
C_{2}=14,320 \\
\mu \mathrm{rd}\end{array}$} & \multicolumn{2}{|c|}{$\begin{array}{l}\text { Trilinear coordinates of } \\
\text { Planckian locus }\end{array}$} & \multirow{2}{*}{$\begin{array}{l}\text { Reciprocal of } \\
\text { the slopes of } \\
\text { the isotemper } \\
\text { ature lines }\end{array}$} \\
\hline & $x$ & $y$ & \\
\hline $\begin{array}{r}0 \\
20 \\
40 \\
60 \\
80\end{array}$ & $\begin{array}{l}0.2399 \\
.2458 \\
.2525 \\
.2605 \\
.2693\end{array}$ & $\begin{array}{r}0.2342 \\
.2429 \\
.2524 \\
.2629 \\
.2743\end{array}$ & $\begin{array}{l}-0.672 \\
-.611 \\
-.536 \\
=.450 \\
-.359\end{array}$ \\
\hline $\begin{array}{l}100 \\
120 \\
140 \\
160 \\
180\end{array}$ & $\begin{array}{l}.2794 \\
.2909 \\
.3037 \\
.3171 \\
.3306\end{array}$ & $\begin{array}{l}.2869 \\
.3003 \\
.3141 \\
.3272 \\
.3396\end{array}$ & $\begin{array}{l}-.266 \\
-.175 \\
-.089 \\
-.012 \\
+.057\end{array}$ \\
\hline $\begin{array}{l}200 \\
220 \\
240 \\
260 \\
280\end{array}$ & $\begin{array}{l}.3445 \\
.3583 \\
.3723 \\
.3865 \\
.4006\end{array}$ & $\begin{array}{l}.3512 \\
.3619 \\
.3717 \\
.3805 \\
.3883\end{array}$ & $\begin{array}{l}+.120 \\
+.175 \\
+.227 \\
+.275 \\
+.319\end{array}$ \\
\hline $\begin{array}{l}300 \\
320 \\
340 \\
360 \\
380\end{array}$ & $\begin{array}{l}.4142 \\
.4273 \\
.4403 \\
.4526 \\
.4646\end{array}$ & $\begin{array}{l}.3950 \\
.4006 \\
.4052 \\
.4089 \\
.4117\end{array}$ & $\begin{array}{l}+.361 \\
+.400 \\
+.437 \\
+.472 \\
+.505\end{array}$ \\
\hline $\begin{array}{l}400 \\
420 \\
440 \\
460 \\
480\end{array}$ & $\begin{array}{l}.4760 \\
.4868 \\
.4973 \\
.5072 \\
.5167\end{array}$ & $\begin{array}{l}.4136 \\
.4148 \\
.4153 \\
.4152 \\
.4146\end{array}$ & $\begin{array}{l}+.535 \\
+.563 \\
+.590 \\
+.615 \\
+.639\end{array}$ \\
\hline $\begin{array}{l}500 \\
550\end{array}$ & $\begin{array}{l}.5256 \\
.5460\end{array}$ & $\begin{array}{l}.4136 \\
.4093\end{array}$ & $\begin{array}{l}+.661 \\
+.713\end{array}$ \\
\hline $\begin{array}{l}600 \\
650\end{array}$ & $\begin{array}{l}.5638 \\
.5797\end{array}$ & $\begin{array}{l}.4033 \\
.3962\end{array}$ & $\begin{array}{l}+.762 \\
+.808\end{array}$ \\
\hline 700 & .5941 & .3886 & +.849 \\
\hline 800 & .6183 & .3731 & +.923 \\
\hline 900 & .6370 & .3587 & +.983 \\
\hline 1000 & .6521 & .3451 & +1.036 \\
\hline
\end{tabular}

normals (iso-temperature lines) nearest color temperature estimated

\footnotetext{
6 H. J. McNicholas, Color and spectral transmittance of vegetzble oils, J. Research NBS 15, 99 (1935) RP815; also Oil \& Soap 12, 167 (1935); see his figure 8.

7 Figure 2 is a reproduction of a figure previously used (see footnote 1, p. 771). Since that time a new color-temperature scale has been adopted (see footnote 9, p. 778) and coincidentally the value of $C_{2}$ used was changed from 14350 to 14320 micron degrees to conform with the International Temperature Scale. This change in $C_{2}$ makes only 0.2-percent change in the temperature (or reciprocal temperature) and is too small to be shown in figure 2. For this reason the old diagram has been used. In figure 3, however, the change (about $0.8 \mu \mathrm{rd}$ ) could be easily seen.

In table 2, the values of color temperature listed were taken from Davis' paper in which the older value of 14350 was used for $\mathrm{C}_{2}$.
} 
according to the UCS system may be approximately read from specifications in the ICI system. Figure 3 shows a small section of figure 2 to an enlarged scale with one normal drawn in for each microreciprocal-degree ( $\mu \mathrm{rd}$ ); as pointed out by Priest, ${ }^{8}$ who proposed the use of reciprocal color temperature, "a difference of one micro-reciprocaldegree is fairly representative of the doubtfully perceptible difference in chromaticity under the most favorable conditions of observation." The large drawing from which this reproduction was made has been used in connection with a check of the present color-temperature scale ${ }^{9}$ with blue filters whose use resulted in near-Planckian colors. The original drawing was found to reproduce estimates of nearest color temperature found directly from the UCS system with a maximum error less than $0.1 \mu \mathrm{rd}$. It will be noted that the normals extend only over the region immediately in the neighborhood of the Planckian locus. The experimental setting of nearest color temperature when a considerable chromaticity difference exists between the illuminant in question and the Planckian radiator at any temperature becomes increasingly difficult and ambiguous as the chromaticity difference is increased. It is doubtful whether the solution given by the UCS system is valid much further than the normals have been extended on figure 3 ; and figure 2, which shows them extended considerably further, may be criticized because it seems to make the false implication that a definite, unambiguous nearest color temperature can be found experimentally for illuminants having colors very notably nonPlanckian.

TABLE 2.-Comparison of conjunctive wave length referring to nearest color temperature via the UCS system with that referring to Davis' correlated color temperature

\begin{tabular}{|c|c|c|c|}
\hline \multirow[b]{2}{*}{$C_{2}=14,350^{\circ} \mathrm{K}$} & \multirow[b]{2}{*}{$C_{2}=14,320 \mu \mathrm{rd}$} & \multicolumn{2}{|c|}{$\begin{array}{c}\text { Conjunctive wave length, } \\
\lambda_{j} \text {, in } \mathrm{m} \mu\end{array}$} \\
\hline & & $\begin{array}{l}\text { Nearest color } \\
\text { temperature } \\
\text { via UCS } \\
\text { system }\end{array}$ & $\begin{array}{l}\text { Davis corre- } \\
\text { lated color } \\
\text { temperature }\end{array}$ \\
\hline $\begin{array}{l}1,803 \\
2,011 \\
2,532 \\
3,053 \\
3,585\end{array}$ & $\begin{array}{l}555.8 \\
498.3 \\
395.8 \\
328.2 \\
279.5\end{array}$ & $\begin{array}{l}588.4 \\
585.6 \\
580.2 \\
575.4 \\
570.9\end{array}$ & $\begin{array}{l}586.5 \\
584.2 \\
579.5 \\
575.5 \\
572.9\end{array}$ \\
\hline $\begin{array}{l}4,116 \\
4,663 \\
5,222 \\
5,781 \\
6,359\end{array}$ & $\begin{array}{l}243.5 \\
214.9 \\
191.9 \\
173.3 \\
157.6\end{array}$ & $\begin{array}{l}567.2 \\
563.0 \\
559.1 \\
555.5 \\
549.8\end{array}$ & $\begin{array}{l}570.2 \\
568.8 \\
567.0 \\
565.8 \\
564.8\end{array}$ \\
\hline $\begin{array}{l}6,945 \\
7,549 \\
8,181 \\
8,826 \\
9,483\end{array}$ & $\begin{array}{l}144.3 \\
132.7 \\
122.5 \\
113.5 \\
105.7\end{array}$ & $\begin{array}{l}546.8 \\
542.0 \\
537.7 \\
533.5 \\
530.0\end{array}$ & $\begin{array}{l}564.0 \\
563.2 \\
562.6 \\
562.1 \\
561.4\end{array}$ \\
\hline $\begin{array}{l}10,187 \\
10,876 \\
11,587 \\
13,161 \\
14,817\end{array}$ & $\begin{array}{l}98.4 \\
92.1 \\
86.5 \\
76.1 \\
67.6\end{array}$ & $\begin{array}{l}526.2 \\
524.5 \\
521.7 \\
517.0 \\
514.4\end{array}$ & $\begin{array}{l}561.0 \\
561.2 \\
561.0 \\
560.3 \\
560.3\end{array}$ \\
\hline
\end{tabular}

8 I. G. Priest, $A$ proposed scale for use in specifying the chromaticity of incandescent illuminants and various phases of daylight, J. Opt. Soc. Am. 23, 41 (1933).

$\theta$ H. T. Wensel, D. B. Judd, and Wm. F. Roeser, Establishment of a scale of color temperature, BS J. R日. search 12, 527 (1934) R P677. 
To facilitate the construction of large-scale plots for accurate work such as figure 3 there have been included in table 1 the trilinear coordinates of the Planckian locus on the ICI system for the reciprocal color temperatures represented by the transformed normals on figure 2 , and the reciprocals of the slopes of these transformed normals. The reciprocals of the slopes are given instead of the slopes, themselves, for convenience because, as may be seen from figure 2 , the slope becomes infinite in the neighborhood of $160 \mu \mathrm{rd}$, but is nowhere zero. Interpolation between the values given in table 1 will yield points on the Planckian locus and also the slopes of the corresponding iso-temperature lines.

Table 2 shows a comparison of conjunctive wave length, $\lambda_{j}$, referring to correlated color temperature computed by Davis' empirical method, with that referring to nearest color temperature from the UCS system. The former were taken from Davis' table $6,{ }^{10}$ the latter were found by extending the iso-temperature lines on the original large-scale plot of figure 2 until they cut the spectrum locus. It may be noted that the agreement is good down to $250 \mu \mathrm{rd}$ (up to $4,000^{\circ} \mathrm{K}$ ) but is rather poor at lower reciprocal color temperatures where validity of the UCS system is best established. ${ }^{11}$

Washington, August 28, 1936.

10 BS. J. Research 7, 659 (1931) RP365.

11 J. Research NBS 14, 41 (1935) R P756; J. Opt. Soc. Am. 25, 24 (1935). 